\title{
Correlation between the Position of the Pituitary Stalk as Determined by Diffusion Tensor Imaging and Its Location as Determined at the Time of Surgical Resection of Pitu- itary Adenomas
}

\author{
Fu-yu Wang, ${ }^{*}$ Peng Wang, ${ }^{*}$ Chen-xuan Yang, Tao Zhou, Jin-li Jiang, Xiang-hui Meng \\ Department of Neurosurgery, The First Medical Center, PLA General Hospital, Beijing, China
}

Objective : An important factor during pituitary adenoma surgery is to preserve pituitary stalk (PS) as this plays a role in reduction of the risk of postoperative diabetes insipidus. The hypothalamic-hypophyseal tract (HHT) projects through the PS to the posterior pituitary gland. To reconstruct white matter fiber pathways, methods like diffusion tensor imaging (DTI) tractography have been widely used. In this report we attempted to predict the position of PS using DTI tractography and to assess its intraoperative correlation during surgery of pituitary adenomas.

Methods : DTI tractography was used to tract the HHT in nine patients before craniotomy for pituitary adenomas. The DTI location of the HHT was compared with the PS position identified at the time of surgery. DTI fiber tracking was carried out in nine patients prior to the planned craniotomy for pituitary adenomas. In one patient, the PS could not be identified during the surgery. In the other eight patients, a comparison was made between the location of the HHT identified by DTI and the position of the PS visualized at the time of surgery.

Results : The position of the HHT identified by DTI showed consistency with the intraoperative position of the PS in seven patients (88.9\% concordance).

Conclusion : This study shows that DTI can identify the position of the HHT and thus the position of the PS with a high degree of reliability.

Key Words : Diffusion tensor imaging · Hypothalamohypophyseal tract.

- Received : October 16, 2019 •Revised : December 12, 2019 •Accepted : December 18, 2019

- Address for reprints : Jin-li Jiang

Department of Neurosurgery, The First Medical Center, PLA General Hospital, NO. 28, Fuxing Road, Beijing 100048, China

Tel : +86-10-66938439, +86-1066938342, Fax : +86-10-68150287, E-mail : jiangj|8058@163.com, ORCID : https://orcid.org/0000-0003-3496-5110

\section{Xiang-hui Meng}

Department of Neurosurgery, The First Medical Center, PLA General Hospital, NO. 28, Fuxing Road, Beijing 100048, China

Tel : +86-10-66938439, Fax : +86-10-68150287, E-mail : 18612357799@163.com, ORCID : https://orcid.org/0000-0003-1630-7560

${ }^{*}$ The two authors contributed equally to this work.

This is an Open Access article distributed under the terms of the Creative Commons Attribution Non-Commercial License (http://creativecommons.org/licenses/by-nc/4.0) which permits unrestricted non-commercial use, distribution, and reproduction in any medium, provided the original work is properly cited. 


\section{INTRODUCTION}

Studies have shown that $10-15 \%$ of all intracranial tumors are comprised of pituitary adenomas ${ }^{16,25)}$. The primary method of treatment for symptomatic patients is surgery. Transsphenoidal surgery is considered the first-choice therapy in all cases except for prolactinomas ${ }^{18)}$. For complex, giant pituitary adenomas with extensive suprasellar extension, however, transcranial resection is the preferred surgery ${ }^{12)}$. In other cases, magnetic resonance imaging (MRI) may show a small sella, or define most of the tumor volume as suprasellar, or predict hard, fibrous consistency difficult to resect safely via the transsphenoidal route. In these cases also the transcranial route may be the best surgical approach. In only about 5\% of the cases, the transcranial approach is necessary ${ }^{20)}$. Pituitary stalk (PS) preservation is important during surgery of pituitary adenomas in order to reduce the risk of post-operative diabetes insipidus (DI). The PS usually deviates away from the tumor in microadenomas. In the case of macroadenomas, it is found that the PS longitudinal axis might have the tendency to directly project towards the normal gland ${ }^{10)}$. It is seen that the stalk and/or the anterior lobe may be encased by the tumor in large or giant pituitary multilobular adenomas ${ }^{17)}$. As a result it is often difficult to visualize the PS because of the distortion of its geometry, or due to the fact that it gets displaced or incorporated in the tumor.

The PS contains the hpothalamic-hypophyseal portal system and hypothalamic-hypophyseal tract (HHT). The HHT projects through the PS to the posterior pituitary gland to control the release of vasopressin and oxytocin. Therefore identification of the HHT can assist in finding the PS position. The optimal method to quantitatively assess the white matter pathways is by the advanced MR technique of diffusion tensor imaging (DTI). It has been widely applied to the cranial nerves $^{7)}$, the pyramidal tract ${ }^{11)}$ and the arcuate fasciculus 22 .

In our study, with the help of DTI, we assessed the location of the HHT preoperatively in patients with pituitary adenomas in whom a transcranial surgical approach was planned. We then compared the position of the PS identified at surgery with the location of the HHT determined by DTI tractography.

\section{MATERIALS AND METHODS}

\section{Patient selection}

Approval for this study was obtained from the Ethic Commitee of PLA General Hospital (S2014-096-01). Written informed consent was obtained from all patients. Nine patients with pituitary adenomas from February 1, 2016 to September 30,2016 , were recruited in this study. All patients received the transcranial surgery. The main demographic and clinical characteristics of the patients are listed in Table 1.

\section{Data acquisition}

Prior to the operations, using a 1.5 T MR system (Espree, Siemens Medical Solution, Erlangen, Germany), images were acquired in a diagnostic suite which was described in detail previously $^{9)}$. The protocol and parameters are listed below. T1weighted 3D magnetization prepared rapid acquisition gradient echo (MPRAGE), sequence (repetition time [TR]/echo time [TE], 1650/3.02 ms; slice thickness, $1 \mathrm{~mm}$; field of view, $250 \mathrm{~mm}$; imaging time, 5 minutes and 18 seconds) were obtained. If the lesion enhanced with intravenous contrast, the 3D T1 MPRAGE sequence was repeated after the intravenous administration of $0.2 \mathrm{~mL} / \mathrm{kg}$ body weight of gadopentetate dimeglumine (Magnevist; Schering, Berlin, Germany); T2weighted sequence (TR/TE, 5500/93 ms; slice thickness, $3 \mathrm{~mm}$; field of view, $230 \mathrm{~mm}$; imaging time, 2 minutes and 8 seconds); T2-weighted fluid-attenuated inversion recovery sequence (T2 FLAIR, TR/TE, 9000/84 ms; slice thickness,

Table1. Main demographic and clinical characteristics of the nine patients

\begin{tabular}{lcccccc}
\hline Patient & $\begin{array}{c}\text { Age } \\
\text { (years) }\end{array}$ & Gender & $\begin{array}{c}\text { Size } \\
(\mathbf{m m})\end{array}$ & PS & GTR & DI \\
\hline 1 & 44 & F & 27 & Yes & Yes & No \\
\hline 2 & 36 & M & 31 & Yes & Yes & No \\
\hline 3 & 53 & M & 47 & Yes & Yes & Yes \\
\hline 4 & 28 & M & 53 & No & No & No \\
\hline 5 & 47 & F & 64 & Yes & Yes & Yes \\
\hline 6 & 51 & M & 45 & Yes & Yes & Yes \\
\hline 7 & 56 & M & 42 & Yes & Yes & Yes \\
\hline 8 & 35 & F & 40 & Yes & Yes & No \\
\hline 9 & 42 & F & 52 & Yes & Yes & Yes \\
\hline PS: & & & & & &
\end{tabular}

PS : pituitary stalk in operation, GTR : gross total resection, DI : new diabetes insipidus, $\mathrm{F}$ : female, $\mathrm{M}$ : male 
$3 \mathrm{~mm}$; field of view, $230 \mathrm{~mm}$; imaging time, 4 minutes and 14 seconds). DTI echo planar (TR/TE, 9400/147 ms; slice thickness, $3 \mathrm{~mm}$; field of view, $250 \mathrm{~mm}$; 12 gradient directions; imaging time, 10 minutes and 22 seconds) was used for DTI-based tractography. During craniotomy the position of the PS was recorded by way of videotaping using the operating microscope.

\section{Data processing}

HHT delineation was carried out by the "fiber tracking" module of iPlan 3.0 software (BrainLab, Feldkirchen, Germany) after acquisition and rigid registration of various image sets. The tractography was carried out with multiple regions of interest (ROI) : the first ROI was positioned at the base of tumor, and the other was positioned between the hypothalamus and upper part of the tumor (Fig. 1) ${ }^{29)}$. The tracking parameters were as follows : fractional anisotropy (FA) threshold value 0.02 ; angle threshold $30^{\circ}$; and shortest fiber bundle length $5 \mathrm{~mm}$. These parameters were utilized to track the fiber bundles across the 2 ROIs concurrently. Tracking of the fiber bundles appeared as mixed redundant fiber bundles and so the tracts were suitably trimmed as per the anatomical knowledge of the operator. The development of 3D anatomical imaging of tumors was as per the normal or enhanced sequences of T1-weighted images. The anatomical and tracking results were fused to determine the course of the HHT in the axial and coronal planes. Sometimes the colors of the tracts were too slight to identify, so the fiber contour was delineated in the "3D fiber objects" module. The properties of the tracts were acquired through the module "plan content", including the numbers of tracts, the minimum FA, maximum FA, average FA, minimum length, maximum length, and average length. Tumor contour was delineated as per the anatomical images in the "object creation" module. On selection of bor- ders, generation of a 3D object was automatically done by covering each structure with a contour. When the tumor contour and HHT contour were fused, the relationship between the tumor and the HHT was clearly detected.

\section{Statistical analysis}

SPSS ver. 19.0 (SPSS Inc., Chicago, IL, USA) was used for statistical data analysis. The correlation was considered significant when the confidence level (two-tailed) was less than or equal to 0.05 .

\section{RESULTS}

The mean patient age was $43.56 \pm 9.26$ years. The participants consisted of five males and four females whose main clinical symptoms were reduction of visual acuity, dizziness, headaches and DI. The disease course ranged from 10 days to 3 years (mean, 1.3 years) (Table 1 ).

All nine patients were treated with a transcranial approach and in eight patients a total resection was achieved while one patient had a subtotal resection (Table 1). Preservation of the PS was achieved in six patients, partial preservation of was achieved in two patients, and thee PS was not detected in one patient. New postoperative DI developed in five patients. The PS location determined during the surgery was used as a basis to compare the HTT location found with DTI.

Data processing of the preoperative MRI data was done with iPlan 3.0 software (BrainLab) for the nine patients. A single experienced neurosurgeon carried out both the fiber tracking and 3D visualization of the HHT. For all the patients, successful tracking and reconstruction was done for the neural fasciculus courses that simultaneously passed through 2 ROIs. Comparison between the locations of the HHTs deter-
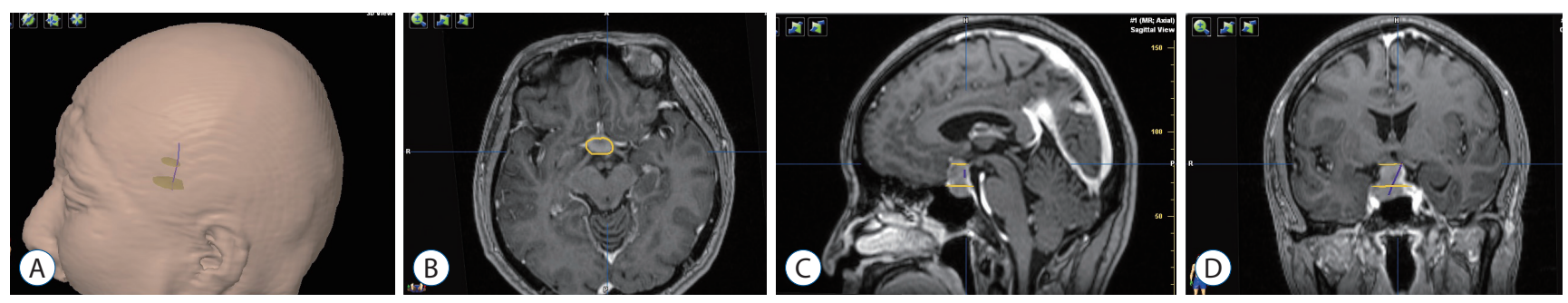

Fig. 1. HHT visualization in a patient with pituitary adenoma. A : The contour of the head with 2 ROls and HHT. B : The yellow contour of the adenoma in the axial plane. C : Two yellow planes show 2 ROI masks. D : HHT after proper pruning ${ }^{29}$. HHT : hypothalamic-hypophyseal tract, ROI : regions of interest. 
mined by DTI and the positions of the PSs identified at surgery were carried out in eight patients. The PS was not detected at surgery in one patient. In seven of eight patients (87.5\%) the HHT visualized by DTI and the position of the PS determined at surgery were identical. Tabulation of the tract properties are seen in Table 2. The mean number of tracts was 2.67 \pm 3.82 (from 1 to 10 ), the mean FA (average FA) was $0.14 \pm 0.06$ (from 0.07 to 0.22 ), and the mean length (average length) was $30.22 \pm 5.73 \mathrm{~mm}$ (from $18 \mathrm{~mm}$ to $37 \mathrm{~mm}$ ).

We made statistical correlation analysis of four variables (tumor size, mean number of tracts, mean FA, and mean length). The correlation is not significant between four variables $(p>0.05)$.

\section{Cases illustration}

\section{Case 1}

A 44-year-old female claimed to suffer from dizzy spells for duration of 10 days. An intrasellar and suprasellar lesion was observed in the MRI (Fig. 2). Because the sella was small, and the patient's visual acuity was poor in the left eye, a left transsylvian fissure approach was performed. The tumor was removed totally, and the PS remained intact. Posterolateral displacement of the PS was noted to the left side. The tumor exhibited gross-total resection with an anatomically intact stalk as seen in the postoperative MRIs. Position of the HHT determined by DTI (Fig. 2A and B) was consistent with the location of the PS determined at surgery (Fig. 2C).

\section{Case 2}

A 36-year-old male presented with decreased visual acuity for 1 year after transsphenoidal resection of a pituitary adenoma 3 years previously. An intrasellar and suprasellar lesion was noted in the MRI (Fig. 3). At the time of the first surgery the tumor was hard to resect, so only partial resection was achieved. The visual acuity was poor in the left eye when ex-

Table 2. Properties of tracts with DTI source in patients with pituitary adenoma

\begin{tabular}{|c|c|c|c|c|c|c|c|}
\hline Patient & No. of tracts & Min. FA & Max. FA & Aver. FA & $\begin{array}{l}\text { Min. length } \\
(\mathrm{mm})\end{array}$ & $\begin{array}{l}\text { Max. length } \\
(\mathrm{mm})\end{array}$ & $\begin{array}{l}\text { Aver. length } \\
(\mathrm{mm})\end{array}$ \\
\hline 1 & 1 & 0.07 & 0.52 & 0.22 & 28 & 28 & 28 \\
\hline 2 & 1 & 0.02 & 0.66 & 0.18 & 31 & 31 & 31 \\
\hline 3 & 1 & 0.04 & 0.09 & 0.07 & 18 & 18 & 18 \\
\hline 4 & 5 & 0.03 & 0.17 & 0.10 & 29 & 31 & 30 \\
\hline 5 & 1 & 0.06 & 0.18 & 0.11 & 35 & 35 & 35 \\
\hline 6 & 3 & 0.03 & 0.40 & 0.08 & 35 & 39 & 37 \\
\hline 7 & 1 & 0.02 & 0.39 & 0.13 & 26 & 26 & 26 \\
\hline 8 & 10 & 0.08 & 0.45 & 0.21 & 31 & 38 & 34 \\
\hline 9 & 1 & 0.03 & 0.56 & 0.17 & 33 & 33 & 33 \\
\hline
\end{tabular}

DTI : diffusion tensor imaging, Min. : minimum, FA : fractional anisotropy, Max. : maximum, Aver. : average
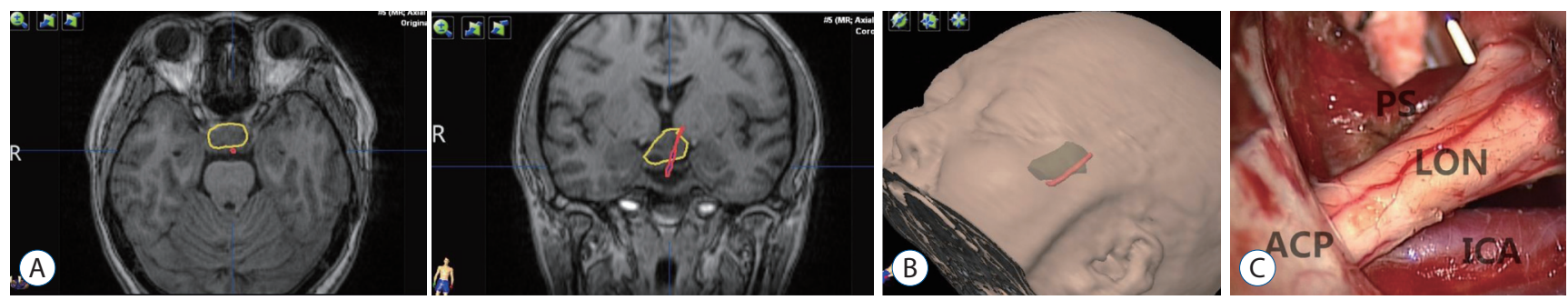

Fig. 2. HHT visualization in case 1. A : Red contour of the HHT and yellow contour of the adenoma. $B$ : The green translucent part shows a $3 \mathrm{D}$ anatomical model of the tumor, and the red part shows the contour of the tracts. C : Intraoperative view of pituitary stalk using the operating microscope. Position of the tracts was consistent with the intraoperative view of the pituitary stalk. PS : pituitary stalk, LON : left optic nerve, ACP : anterior clinoid process, ICA : internal carotid artery, $\mathrm{HHT}$ : hypothalamic-hypophyseal tract. 

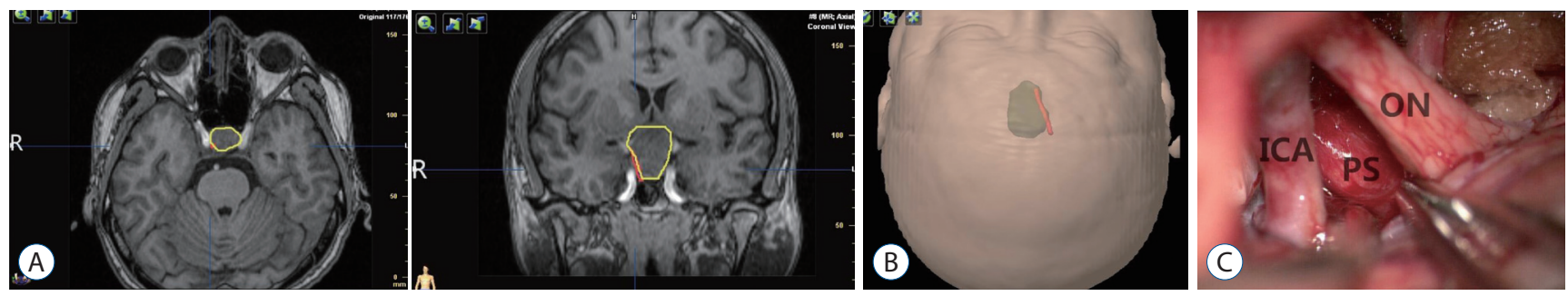

Fig. 3. HHT visualization in case 2. A : Red contour of the HHT and yellow contour of the pituitary adenoma. B : The green translucent part is a 3D anatomical model of the tumor, and the red part shows the contour of tracts. C: Visualization of the pituitary stalk at the time of surgery. Position of the tracts was consistent with the intraoperative observations of the position of the pituitary stalk. ON : optic nerve, ICA : internal carotid artery, PS : pituitary stalk, $\mathrm{HHT}$ : hypothalamic-hypophyseal tract.
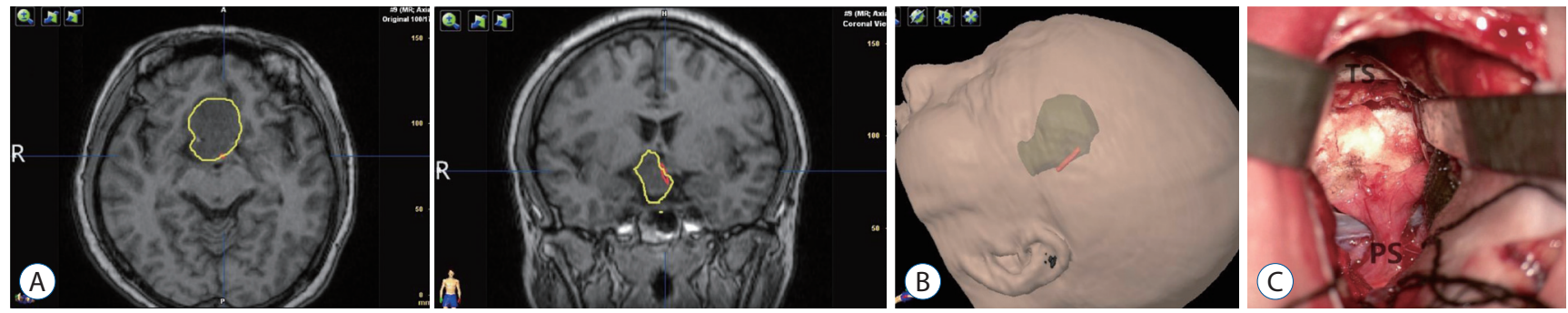

Fig. 4. HHT visualization in case 3. A : Red contour of the HHT and yellow contour of the adenoma. B : The green translucent part shows a 3D anatomical model of the tumor, and the red part shows the contour of the tracts. C : Intra-operative location of the pituitary stalk. Position of the tracts was consistent with intraoperative observations of the location of the pituitary stalk. TS : tuberculum sellae, PS : pituitary stalk, HHT : hypothalamichypophyseal tract.

amination, therefore we used the left trans-sylvian fissure approach. The tumor was resected completely, and the PS was intact and pushed posterolaterally to the right side. From postoperative MRIs taken, it was found that there was grosstotal resection of the tumor and that the stalk was anatomically intact. The position of the HHT determined by DTI (Fig. 3A and B) was consistent with the position of the PS determined at surgery (Fig. 3C).

\section{Case 3}

A 53-year-old female presented with decreased visual acuity for 3 years and progressive deterioration in the right eye for half a year. From the MRI, the presence of intrasellar and suprasellar lesion with extension to anterior cranial base was noted (Fig. 4). The patient underwent trans-anterior longitudinal fissure approach surgery. The tumor was removed completely, and the PS was intact. There was posterolateral displacement to the left side noted. Gross-total resection of the tumor as well as an anatomically intact stalk was noticed in postoperative MRIs. Position of the HHT determined by DTI (Fig. 4A and B) was consistent with the intraoperative observations of the position of the PS (Fig. 4C).

\section{Case 4}

A 28-year-old female presented with decreased visual acuity for 4 years and blindness in the right eye for 1 month. From the MRI, an intrasellar and suprasellar lesion with extension to the third ventricle was identified (Fig. 5). The patient underwent craniotomy via a right frontal temporal approach. During the surgery, the tumor tissue was hard to resect, and the anatomical relationships between the tumor and normal structures was not clear, so only a subtotal resection was accomplished. The PS could not be identified. Postoperative MRI demonstrated residual tumor. The position of HHTs was identical in the preoperative and postoperative images (Fig. 5B).

\section{DISCUSSION}

Surgery remains the most important treatment for PA, particularly for nonfunctional pituitary macroadenomas ${ }^{1,2}$. Patients with injured PS present with more endocrine dysfunction postoperatively. It is important to detect and protect PS during surgery. Even partial preservation of the PS has a vital role in the secretion of antidiuretic hormone postoperatively. 


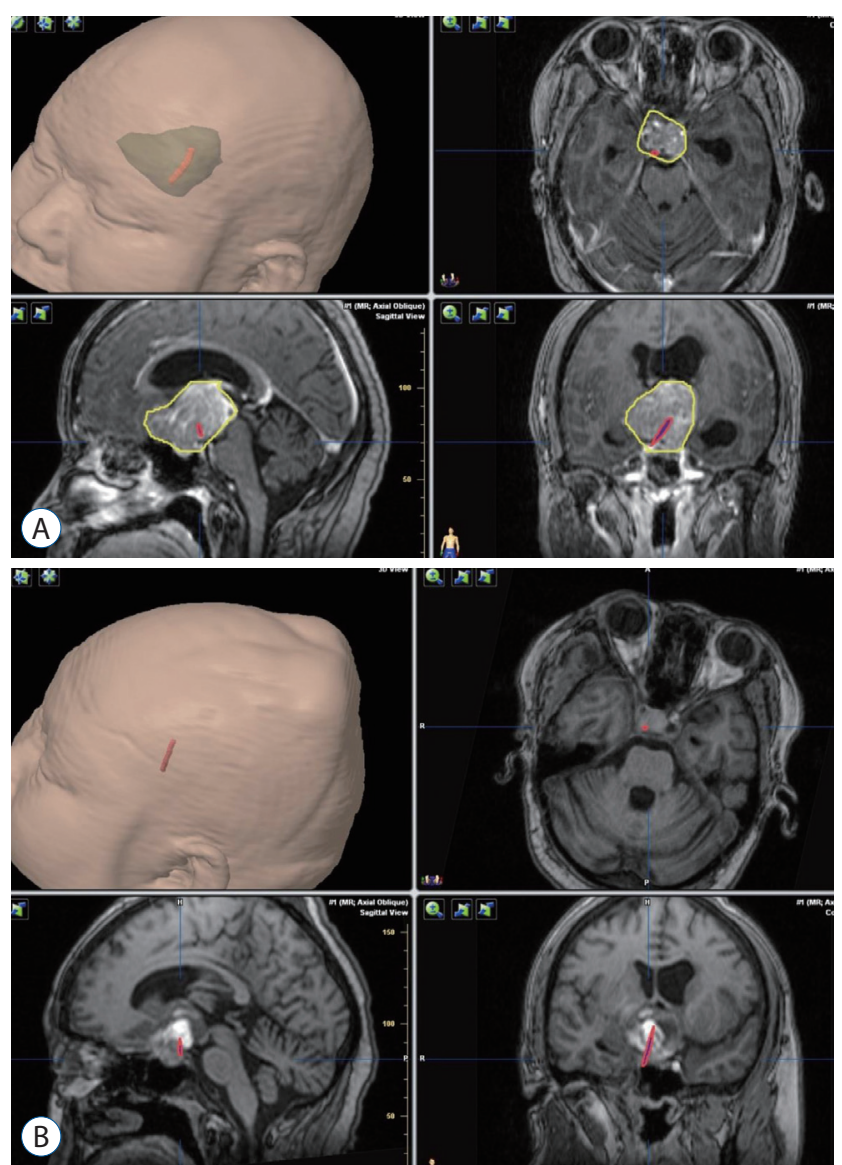

Fig. 5. HHT visualization in case 4. A : The yellow translucent part shows a 3D anatomical model of the tumor, and the red part shows the contour of tracts. The tracts were encased by the tumor. B : Postoperative MRI demonstrated residual tumor. The red part shows the contour of tracts. The tracts were encased by the residual tumor. HHT : hypothalamichypophyseal tract, MRI : magnetic resonance imaging.

Damage to the PS during transcranial surgery of pituitary tumors usually leads of post-operative DI due to injury of the HHT, or HHP. Pre-operative identification of the position of the PS might reduce the incidence of injury to the tract and thus reduce the incidence of DI.

Ammirati et al. ${ }^{3)}$ conducted a meta-analysis study which indicated that out of the patients who underwent pituitary surgery, $11.6 \%$ suffered from postoperative hypopituitarism and permanent DI was recorded in $4.3 \%$. The median eminence of the hypothalamus is connected to the pituitary gland by the funnel-like PS. Numerous MRI studies with regards to the normal PS are available. Simmons et al. ${ }^{26)}$ showed that the signal intensity of the PS on unenhanced T1-weighted images was less than that of the signal intensity of the neurohypophysis and the optic chiasm. After the injection of contrast mate- rial, the PS was enhanced. For normal subjects of all ages, Araki et al. ${ }^{4}$ described high signal intensity of the PS on FLAIR MR images. Out of 29 patients, it was found that for 20 (69\%), the T2 MR images of the PS showed central hyperintensity with a peripheral rim of iso-intensity. A homogeneous iso-intensity was seen for the remaining nine patients ${ }^{24)}$.

Visualization of the HHT is problematic with normal MRI techniques. Among many MRI techniques, DTI can quantitatively make assessments of white matter integrity. DTI has wide application in the assessment of the white matter $\operatorname{tracts}^{11,23)}$, cranial nerve $\mathrm{e}^{7)}$ and some brain diseases ${ }^{19,32)}$. FA is one of most important DTI parameters, and FA value is a reflection of the alignment degree of cellular structures and their structural integrity ${ }^{6}$. However, few reports about the DTI method on the HHT are presented so far. This report represents the first attempt to use DTI fiber tracking to identify and locate the HHT.

In our study, we successfully identified the HHT in all patients with DTI fiber tracking. Comparison between the HHT visualization by DTI and the intraoperative location of PS was made in eight patients. The PS was not detected in one patient. There was consistency between the HHT visualization by DTI and the intraoperative location of PS in seven of eight patients (87.5\%). The possible reasons why the DTI and PS positions did not correlate in one patient were complicated. It was low FA threshold value, which led to incorrect track of some nonfiber bundle structures and the deviation in visualizing the course of the fiber bundles. For future studies, it is to obtain the better results by choosing precise ROIs and with adjustment of the FA threshold value ${ }^{211}$. The main drawback of the DTI tractography is the inadequate spatial resolution and the poor signal-to-noise ratio $(\mathrm{SNR})^{23)}$. The application of large voxel sizes led to more partial volume averaging and contamination from adjacent fiber bundles. Low SNR led to overestimating of anisotropy and more inaccuracies in eigenvector estimation, which increases erroneous white matter connections and false positive results ${ }^{14}$. For further studies, the use of higher spatial resolution might decrease the obstacles from crossing fibers and susceptibility artifacts ${ }^{15}$.

Optimal surgical outcomes for pituitary adenomas provide maximal tumor resection and good preservation of PS. During surgery, a variety of methods may be used to identify the PS. The first is to confirm the PS by visualizing the structure going via the diaphragm sellae and connecting the hypothala- 
mus and pituitary gland. Secondly, is to locate the PS by identifying the longitudinal stria medullaris structures on the surface of that structure ${ }^{30)}$. Thirdly, is to locate the PS by reference to the location of the anterior commissure ${ }^{28)}$. However, in large or giant pituitary adenomas the tumor can encase or invade the stalk, as observed in case 4 . Under these conditions, it is difficult to protect the PS. It may be helpful for surgeons for better identification and preservation of the PS by acquiring information about the location of HHT by way of the DTI fiber tracking technique. In this study, the HHT tract location was consistent with the original PS location in seven of eight cases (87.5\%). For further studies, implementation of higher spatial resolution MRI techniques might improve the results.

In this study, we also revealed the ability to evaluate the tract numbers, FA values and length. In nine cases, the results indicated properties of the tracts. It was seen that the tract number was comparably low with, only one tract seen in six patients, three in one patients, five in one patient, 10 in one patient. No correlation between the size of tumor and number of tract was found. No correlation between the size of tumor and mean FA and mean length was found. The reasons were complicated. The HHT may be damaged by the tumor, so fiber tracking could be compromised in the fiber-tracking algorithm. The interference of cerebrospinal fluid would influence the resolution of the DTI signals, which would cause a significant reduction of the number of tracts. The mean FA observed in our study was $0.14 \pm 0.06$ which was less that other cranial nerves $^{8)}$ and white matter tracts ${ }^{5,31)}$. The reasons might be that the HHT was unmyelinated fiber ${ }^{13,27)}$, and the compression of tumor might result in decreased FA value ${ }^{31)}$. The mean length of tract was $30.22 \pm 5.73 \mathrm{~mm}$, longer than the normal length of normal $\mathrm{PS}^{24)}$. The results were reasonable, because in normal state, the HHT produced from paraventricular and the supraoptic nuclei in hypothalamus went through the PS to the posterior lobe, the length was longer than the PS. Moreover, stretching of HTT due to the tumor was noticed in pathological conditions.

There are some limitations in this study. The number of patients used in this study is not enough to arrive at a more comprehensive quantitative evaluation of the effects of tumor features. Therefore, more cases include different pathologies around the sellare will be needed to demonstrate the association between DTI tractography and characteristics of tumors.

\section{CONCLUSION}

Our study demonstrated that HHT can be successfully identified by DTI fiber tracking techniques and that there is a strong correlation between the HHT location and the PS location identified at the time of surgical resection of pituitary adenomas. Further study is required to determine whether preoperative identification of the HHT by DTI can reduce the incidence of DI due to injury to the PS at the time of surgical treatment of pituitary adenomas.

\section{CONFLICTS OF INTEREST}

No potential conflict of interest relevant to this article was reported.

\section{INFORMED CONSENT}

Informed consent was obtained from all individual participants included in this study.

\section{AUTHOR CONTRIBUTIONS}

\author{
Conceptualization : JJ, XM \\ Data curation : TZ \\ Formal analysis : $\mathrm{PW}$ \\ Methodology : CY \\ Project administration : JJ \\ Visualization : TZ \\ Writing - original draft : FW, PW \\ Writing - review \& editing: XM
}

\section{ORCID}

$\begin{array}{ll}\text { Fu-yu Wang } & \text { https://orcid.org/0000-0003-2232-5423 } \\ \text { Peng Wang } & \text { https://orcid.org/0000-0002-0837-6382 } \\ \text { Chen-xuan Yang } & \text { https://orcid.org/0000-0002-3830-7169 } \\ \text { Tao Zhou } & \text { https://orcid.org/0000-0002-4273-5262 } \\ \text { Jin-li Jiang } & \text { https://orcid.org/0000-0003-3496-5110 } \\ \text { Xiang-hui Meng } & \text { https://orcid.org/0000-0003-1630-7560 }\end{array}$




\section{References}

1. Ahn JY, Jung JY, Kim J, Lee KS,Kim SH : How to overcome the limitations to determine the resection margin of pituitary tumours with lowfield intra-operative MRI during trans-sphenoidal surgery: usefulness of Gadolinium-soaked cotton pledgets. Acta Neurochir (Wien) 150 : 763-771; discussion 771, 2008

2. Albayrak B, Samdani AF, Black PM : Intra-operative magnetic resonance imaging in neurosurgery. Acta Neurochir (Wien) 146 : 543-556; discussion 557, 2004

3. Ammirati $M$, Wei $L$, Ciric I : Short-term outcome of endoscopic versus microscopic pituitary adenoma surgery: a systematic review and metaanalysis. J Neurol Neurosurg Psychiatry 84 : 843-849, 2013

4. Araki Y, Ashikaga R, Takahashi S, Ueda J, Ishida 0 : High signal intensity of the infundibular stalk on fluid-attenuated inversion recovery MR.

AJNR Am J Neuroradiol 18 : 89-93, 1997

5. Banaszek A, Bladowska J, Pokryszko-Dragan A, Podemski R, Sąsiadek MJ : Evaluation of the degradation of the selected projectile, commissural and association white matter tracts within normal appearing white matter in patients with multiple sclerosis using diffusion tensor MR imaging - a preliminary Study. Pol J Radiol 80 : 457-463, 2015

6. Basser PJ, Pierpaoli C : Microstructural and physiological features of tissues elucidated by quantitative-diffusion-tensor MRI. J Magn Reson B 111 : 209-219, 1996

7. Borkar SA, Garg A, Mankotia DS, Joseph SL, Suri A, Kumar R, et al. : Prediction of facial nerve position in large vestibular schwannomas using diffusion tensor imaging tractography and its intraoperative correlation.

Neurol India 64 : 965-970, 2016

8. Chen F, Chen L, Li W, Li L, Xu X, Li W, et al. : Pre-operative declining proportion of fractional anisotropy of trigeminal nerve is correlated with the outcome of micro-vascular decompression surgery. BMC Neurol 16 : 106, 2016

9. Chen X, Xu BN, Meng X, Zhang J, Yu X, Zhou D : Dual-room 1.5-T intraoperative magnetic resonance imaging suite with a movable magnet: implementation and preliminary experience. Neurosurg Rev 35 : 95109; discussion 109-110, 2012

10. Cho CH, Barkhoudarian G, Hsu L, Bi WL, Zamani AA, Laws ER : Magnetic resonance imaging validation of pituitary gland compression and distortion by typical sellar pathology. J Neurosurg 119 : 1461-1466, 2013

11. George E, Heier L, Kovanlikaya I, Greenfield J : Diffusion tensor imaging of pyramidal tract reorganization after pediatric stroke. Childs Nerv Syst 30 : 1135-1139, 2014

12. Guo F, Song L, Bai J, Zhao P, Sun H, Liu X, et al. : Successful treatment for giant pituitary adenomas through diverse transcranial approaches in a series of 15 consecutive patients. Clin Neurol Neurosurg 114 : 885-890, 2012

13. Harris GW, Manabe $Y$, Ruf $K B$ : A study of the parameters of electrical stimulation of unmyelinated fibres in the pituitary stalk. J Physiol 203 : 67-81, 1969

14. Hasan KM, Kamali A, Kramer LA : Mapping the human brain white mat- ter tracts relative to cortical and deep gray matter using diffusion tensor imaging at high spatial resolution. Magn Reson Imaging 27 : 631 636, 2009

15. Kamali A, Hasan KM, Adapa P, Razmandi A, Keser Z, Lincoln J, et al. : Distinguishing and quantification of the human visual pathways using high spatial resolution diffusion tensor tractography. Magn Reson Imaging 32 : 796-803, 2014

16. Kovacs K, Horvath E : Pathology of pituitary tumors. Endocrinol Metab Clin North Am 16 : 529-551, 1987

17. Li HY, Feng CY, Zhang C, Su J, Yuan J, Xie Y, et al. : Microscopic surgery for pituitary adenomas to preserve the pituitary gland and stalk. Exp Ther Med 13 : 1011-1016, 2017

18. Loyo-Varela M, Herrada-Pineda T, Revilla-Pacheco F, Manrique-Guzman $S$ : Pituitary tumor surgery: review of 3004 cases. World Neurosurg $79:$ :331-336, 2013

19. Ontaneda D, Sakaie K, Lin J, Wang X, Lowe MJ, Phillips MD, et al. : Identifying the start of multiple sclerosis injury: a serial DTI study. J Neuroimaging 24 : 569-576, 2014

20. Pratheesh R, Rajaratnam S, Prabhu K, Mani SE, Chacko G, Chacko AG : The current role of transcranial surgery in the management of pituitary adenomas. Pituitary $16:$ 419-434, 2013

21. Prieto R, Pascual JM, Rosdolsky M, Castro-Dufourny I, Carrasco R, Strauss $S$, et al. : Craniopharyngioma adherence: a comprehensive topographical categorization and outcome-related risk stratification model based on the methodical examination of 500 tumors. Neurosurg Focus 41 : E13, 2016

22. Psomiades M, Fonteneau C, Mondino M, Luck D, Haesebaert F, SuaudChagny MF, et al. : Integrity of the arcuate fasciculus in patients with schizophrenia with auditory verbal hallucinations: a DTI-tractography study. Neuroimage Clin 12 : 970-975, 2016

23. Salmela MB, Cauley KA, Nickerson JP, Koski CJ, Filippi CG : Magnetic resonance diffusion tensor imaging (MRDTI) and tractography in children with septo-optic dysplasia. Pediatr Radiol 40 : 708-713, 2010

24. Satogami N, Miki Y, Koyama T, Kataoka M, Togashi K : Togashi normal pituitary stalk: high-resolution MR imaging at 3T. AJNR Am J Neuroradiol $31: 355-359,2010$

25. Scheithauer BW : Surgical pathology of the pituitary: the adenomas. Part I. Pathol Annu 19 Pt 1 : 317-374,1984

26. Simmons GE, Suchnicki JE, Rak KM, Damiano TR : MR imaging of the pituitary stalk: size, shape, and enhancement pattern. AJR Am J Roentgenol 159 : 375-377, 1992

27. Stone BS, Zhang J, Mack DW, Mori S, Martin LJ, Northington FJ : Delayed neural network degeneration after neonatal hypoxia-ischemia. Ann Neurol 64 : 535-546, 2008

28. Sui M, Liu S, Liu M, Li Y, Tian Y : Locating of the pituitary stalk for craniopharyngioma surgery of transfrontobasal interhemispheric approach. J Craniofac Surg 24 : 2106-2109, 2013

29. Wang F, Zhang J, Wang P, Zhou T, Meng X, Jiang J : Prediction of pituitary stalk position in pituitary adenomas by visualization of the hypothalamo-hypophyseal tract using diffusion tensor imaging tractography. Medicine (Baltimore) 97 : e0052, 2018 
30. Xiao G, Yuan X, Yuan J, Krumtally NA, Li Y, Feng C, et al. : Pituitary stalk management during the microsurgery of craniopharyngiomas. Exp Ther Med 7 : 1055-1064, 2014

31. Yao Y, Ulrich NH, Guggenberger R, Alzarhani YA, Bertalanffy H, Kollias SS : Quantification of corticospinal tracts with diffusion tensor imaging in brainstem surgery: prognostic value in 14 consecutive cases at 3T magnetic resonance imaging. World Neurosurg 83 : 1006-1014, 2015

32. Zheng Z, Shemmassian S, Wijekoon C, Kim W, Bookheimer SY, Pouratian N : DTI correlates of distinct cognitive impairments in Parkinson's disease. Hum Brain Mapp 35 : 1325-1333, 2014 